\title{
DEFINING THE BOUNDARY OF A HOMOLOGY MANIFOLD
}

\author{
W.J.R. MITCHELL
}

(Communicated by Frederick R. Cohen)

\begin{abstract}
We show that the natural homological analogue of the definition of topological manifold with boundary does indeed properly capture the properties required of a homology manifold with boundary. Accordingly existing definitions requiring that the boundary is already a (homology) manifold can be simplified.
\end{abstract}

A topological $n$-manifold with boundary $M$ is normally defined to be a space such that for each point $m$ there exists an embedding $h:\left(B^{n}, p\right) \rightarrow(M, m)$ with $h\left(B^{n}\right)$ a neighborhood of $h(p)=m ; p$ may lie anywhere in the standard $n$-ball $B^{n}$, and the boundary is defined as the set of $m$ for which $p$ lies in $\partial B^{n}=\{x \mid\|x\|=1\}$. Of course, this begs a question as to whether the boundary is well defined, or in other words, whether every self-homeomorphism of $B^{n}$ carries $\partial B^{n}$ to itself. As every graduate student knows, the solution of this difficulty is nontrivial and requires a proof of invariance of domain, the JordanBrouwer separation theorem, or equivalent. From this it also follows that the boundary is itself a manifold of dimension $n-1$ and with empty boundary.

How might this be generalised to homology manifolds? A straightforward translation to homological language of the existence of a local homeomorphism with the compact finite-dimensional space $B^{n}$ suggests the following definition.

Definition. Let $X$ be a locally compact Hausdorff space of finite cohomological dimension over some group $G$. Suppose that for all $x \in X, H_{*}(X, X-x ; G) \cong$ $H_{*}\left(B^{n}, B^{n}-p ; G\right)$ for some point $p=p(x) \in B^{n}$, i.e.

(a) $H_{*}(X, X-x ; G) \cong 0$ for $* \neq n$.

(b) $H_{n}(X, X-x ; G) \cong 0$ or $G$.

Then $X$ is said to be a homology n-manifold with boundary over $G$. The boundary $\partial X$ of $X$ is defined by

$$
\partial X=\left\{x \in X \mid H_{n}(X, X-x ; G) \cong 0\right\} .
$$

Received by the editors November 25, 1988.

1980 Mathematics Subject Classification (1985 Revision). Primary 57P05; Secondary 55N35, $55 \mathrm{M} 10$.

Key words and phrases. Cohomological dimension, homology manifold, manifold with boundary, sheaf cohomology, Borel-Moore homology. 
It is automatic that the boundary of $X$ is well defined, but far from obvious that $\partial X$ is itself a homology manifold, or that it has empty boundary. Presumably for this reason, earlier authors adopted definitions of homology manifold with boundary designed to guarantee that $\partial X$ is homology manifold (if they had not followed Wilder, the founding father of the subject, by substantially ignoring manifolds with boundary [Wi]). Raymond and Repovš [Ra, Re] both include in their definitions an insistence that the boundary is already a homology $(n-1)$-manifold. White [Wh] adds a relative homology condition to what is essentially the above definition, easily forcing a similar conclusion.

We show this is unnecessary, in that such requirements follow from the definition stated above.

Theorem. Let $X$ be a homology n-manifold with boundary over the integers $\mathbb{Z}$, and suppose $X$ is first countable. Then either $\partial X$ is empty, or it is a homology $(n-1)$-manifold with empty boundary.

A substantial partial result to this effect is given in a little-known paper of Harlap [Ha]. No apology is made for repeating some of his arguments, since even the experts seem unaware of both his paper and the extension to it that is represented by the above result.

Definitions and notations. Cohomology will be sheaf (= Alexander-Čech) cohomology, while homology will be Borel-Moore, which under the cases under discussion coincides with the homology of Steenrod-Sitnikov-Skljarenko [S]; a general reference for Borel-Moore homology is [B2]. A space has finite cohomological dimension over $G$ if there exists an integer $N$ such that for all open sets $U, H_{c}^{N}(U ; G)=0$, where $H_{c}^{*}$ denotes cohomology with compact supports. A space is cohomologically locally connected over $G$ if for any neighborhood $U$ of any point $x$ in $X$, there exists a smaller neighborhood $V$ of $x$ such that $i^{*}: \widetilde{H}^{*}(U ; G) \rightarrow \widetilde{H}^{*}(V ; G)$ is zero. ( $\widetilde{H}^{*}$ denotes reduced cohomology.) In general we shall take $G$ to be the integers $\mathbb{Z}$ and omit this from notation.

Let $\mathscr{H}_{*}$ denote the homology sheaf of $X$ [B2]. Its stalks are given by $\mathscr{H}_{*, x} \cong$ $H_{*}(X, X-x) \cong \lim _{U}\left(H_{*}(U)\right.$, where the limit runs over open neighborhoods $U$ of $x$. Here $H_{*}$ denotes Borel-Moore homology with closed supports; it is analogous to singular homology based on locally finite chains, and by excision $H_{*}(U) \cong H_{*}^{c}(X, X-U)$, where $H_{*}^{c}$ corresponds to the more familiar singular group based on finite chains.

Throughout let $X$ be a homology $n$-manifold with boundary over $\mathbb{Z}$.

Lemma 1. $X$ is cohomologically locally connected.

Proof. Since the homology sheaf has countable stalks, this follows from [M].

Lemma 2. $\partial X$ is closed and nowhere dense in $X$.

Proof. By the universal coefficient theorem $X$ is a homology $n$-manifold with boundary over $\mathbb{Z}_{2}$ (with the same boundary as over $\mathbb{Z}$ ). If $\partial X=X$, i.e. $\mathscr{H}_{*}=0$ everywhere, then for any open $U$ and any $x \in U$, there exists an open 
$V$ such that $x \in V \subset U$ and $H_{*}\left(U ; \mathbb{Z}_{2}\right) \rightarrow H_{*}\left(V, \mathbb{Z}_{2}\right)$ is zero. (Note that by Lemma 1 , this image is finitely generated for sufficiently small $V$, and so must be zero for possibly smaller $V$ to achieve the correct direct limit.) Thus the dual map $j^{*}: H_{c}^{*}\left(V ; \mathbb{Z}_{2}\right) \rightarrow H_{c}^{*}\left(U ; \mathbb{Z}_{2}\right)$ is also zero. By a well-known MayerVietoris and limiting argument [B2, II.15.13], $H_{c}^{*}\left(U ; \mathbb{Z}_{2}\right)=0$ for all open sets $U$, forcing the absurd conclusion that $\operatorname{dim}_{\mathbb{Z}_{2}} X<n$ for all $n$. Since only $\mathscr{H}_{n}$ can be nonzero, we conclude that for some open $U$ the Borel-Moore homology group $H_{n}\left(U ; \mathbb{Z}_{2}\right)$ is nonzero, and moreover gives rise to a nontrivial section of $\mathscr{H}_{n}$, i.e. $\partial X \neq X$. Let $\sigma$ be a nontrivial section of $\mathscr{H}_{n}$ determined by some nonzero element of some group $H_{n}\left(U ; \mathbb{Z}_{2}\right)$ where $U$ is open. Suppose $A \subset U$ is the support of $\sigma$ (i.e. $A=\{x \in U \mid \sigma(x) \neq 0\}$ ), and if possible, suppose that $A \neq U$. By Lemma 1 there is no loss of generality in assuming $U$ is connected. By $[\mathrm{B} 2, \mathrm{~V} .8]$ there is a spectral sequence

$$
E_{2}^{p, q}=H_{c}^{p}\left(A ; \mathscr{H}_{-q}\left(\mathbb{Z}_{2}\right)\right) \Rightarrow H_{-p-q}^{c}\left(U, U-A ; \mathbb{Z}_{2}\right) .
$$

This gives an isomorphism

$$
H_{c}^{n}\left(A ; \mathbb{Z}_{2}\right) \cong H_{c}^{n}\left(A ; \mathscr{H}_{n}\left(\mathbb{Z}_{2}\right)\right) \cong H_{0}^{c}\left(U, U-A ; \mathbb{Z}_{2}\right) .
$$

If $A \neq U$, the exact sequence of the pair $(U, U-A)$

$$
\cdots \rightarrow H_{0}^{c}\left(U-A ; \mathbb{Z}_{2}\right) \rightarrow H_{0}^{c}\left(U ; \mathbb{Z}_{2}\right) \rightarrow H_{0}^{c}\left(U, U-A ; \mathbb{Z}_{2}\right) \rightarrow 0
$$

shows that $H_{c}^{n}\left(A ; \mathbb{Z}_{2}\right)$, and hence also its dual space $H_{n}\left(A ; \mathbb{Z}_{2}\right)$, must be zero. However it is well known that if (as here ) $\operatorname{dim}_{\mathbb{Z}_{2}} X=n$, then $H_{n}\left(K ; \mathbb{Z}_{2}\right)$ is the module of sections of $\mathscr{H}_{n}\left(\mathbb{Z}_{2}\right)$ over $K$-see for example [S, or Ha, Corollary 7]. Thus to avoid contradiction, we must have $A=U$, and so $\partial X$ is closed. Clearly $\partial X$ is nowhere dense, since if not, then its interior would be a space of finite cohomological dimension but with everywhere zero homology sheaf, leading to a contradiction as before.

Lemma 3. $\mathscr{H}_{*}(X) \mid \partial X=0$ and $\mathscr{H}_{n} \mid X-\partial X$ is locally constant.

Proof. The first assertion is trivial, while the second is Bredon's result [B1], whose proof we used in Lemma 2.

Lemma 4. (a) For any open set $U, H^{*}(U-\partial X) \cong 0$.

(b) For all $x \in \partial X$, there exists a neighborhood $W$ of $x$ in $X$ such that $\mathscr{H}_{n} \mid W-\partial X$ is constant.

For $U$ open in $X$, there again is a spectral sequence [B2, V.8]

$$
E_{2}^{p, q}=H_{c}^{p}\left(\partial X \cap U ; \mathscr{H}_{-q}(U)\right) \Rightarrow H_{-p-q}^{c}(U, U-\partial X) .
$$

This collapses by Lemma 3 to yield $H_{*}^{c}(U, U-\partial X) \cong 0$. Now $U$ and $U-\partial X$ are both open in $X$ and so cohomologically locally connected by Lemma 1 . Hence the universal coefficient theorem [B2, V.12.7].

$$
\begin{aligned}
0 & \rightarrow \operatorname{Ext}\left(H_{*-1}^{c}(U, U-\partial X), \mathbb{Z}\right) \rightarrow H^{*}(U, U-\partial X) \\
& \rightarrow \operatorname{Hom}\left(H_{*}(U, U-\partial X), \mathbb{Z}\right) \rightarrow 0
\end{aligned}
$$

implies that $H^{*}(U, U-\partial X) \cong 0$, proving (a). 
Consider the cohomology sequences (with $\mathbb{Z}_{2}$ coefficients) of the pairs $(V$, $V-\partial X)$ and $(W, W-\partial X)$, where $W$ is chosen for $V$ as in the definition of cohomological connectedness,

$\cdots \rightarrow H^{1}\left(V ; \mathbb{Z}_{2}\right) \rightarrow H^{1}\left(V-\partial X ; \mathbb{Z}_{2}\right) \underset{\downarrow}{\rightarrow} H^{2}\left(V, V-\partial X ; \mathbb{Z}_{2}\right) \rightarrow \cdots$ $\cdots \rightarrow H^{1}\left(W ; \mathbb{Z}_{2}\right) \rightarrow H^{1}\left(W-\partial X ; \mathbb{Z}_{2}\right) \rightarrow H^{2}\left(W, W-\partial X ; \mathbb{Z}_{2}\right) \rightarrow \cdots$. Using (a) and the fact that $i^{*}: H^{1}\left(V ; \mathbb{Z}_{2}\right) \rightarrow H^{1}\left(W ; \mathbb{Z}_{2}\right)$ is zero, we have that $i^{*}: H^{1}\left(V-\partial X ; \mathbb{Z}_{2}\right)-H^{1}\left(W-\partial X ; \mathbb{Z}_{2}\right)$ is zero. Now on $X-\partial X, \mathscr{H}_{n}$ may be considered as a locally constant numerable bundle with group $\mathbb{Z}_{2}=$ $\operatorname{Aut}(\mathbb{Z})$. Thus by $[\mathrm{D}]$ it has a classifying map into $B \mathbb{Z}_{2} \simeq K\left(\mathbb{Z}_{2}, 1\right)$. By [Hu] $H^{k}(Z ; G) \cong[Z, K(G, k)]$ for any metric space $Z$. Since classifying maps are natural with respect to restriction to open subsets, the classifying map for $\mathscr{H}_{n} \mid W-\partial X$ is trivial, and the result follows. (Alternatively one may show more directly that the obstruction to piecing together local trivialisations of the sheaf $\mathscr{H}_{n}$ is a class in $H^{1}\left(W-\partial X ; \mathbb{Z}_{2}\right)$.)

Proof of theorem. If $\partial X \neq \varnothing$, let $x \in \partial X$. Since $U \cap \partial X$ is closed in $U$, we have an exact sequence

$$
\cdots \rightarrow H_{p+1}(U-\partial X) \rightarrow H_{p}(U \cap \partial X) \rightarrow H_{p}(U) \rightarrow \cdots,
$$

where $U$ is an open neighborhood of $x$ in $X$. Taking the direct limit over such $U$ preserves exactness. Now, by hypothesis

$$
\overrightarrow{\lim }_{U}\left(H_{*}(U) \cong H_{*}(X, X-x) \cong 0,\right.
$$

and so

$$
H_{p}(\partial X, \partial X-x) \cong \overrightarrow{\lim }_{U} H_{p}(U \cap \partial X) \cong \overrightarrow{\lim }_{U} H_{p+1}(U-\partial X) .
$$

Now by Lemma 4(b), for sufficiently small open $U$,

$$
H^{n-p-1}(U-\partial X) \cong H^{n-p-1}\left(U-\partial X ; \mathscr{H}_{n}\right) \cong H_{p+1}(U-\partial X),
$$

where the second isomorphism is by duality in the homology manifold $U-\partial X$.

Taking the direct limit of the cohomology sequences of the pairs $(U, U-$ $\partial X)$, and noting that the relative groups vanish by Lemma 4(a), we find that $\overrightarrow{\lim }_{U} H^{n-p-1}(U-\partial X) \cong \overrightarrow{\lim }_{U} H^{n-p-1}(U)$. Now by Lemma 1,

$$
\overrightarrow{\lim }_{U} H^{k}(U) \cong \begin{cases}0, & \text { if } k \neq 0 \\ \mathbb{Z}, & \text { if } k=0 .\end{cases}
$$

Hence

$$
\begin{aligned}
H_{p}(\partial X, \partial X-x) & \cong \lim _{U} H_{p+1}(U-\partial X) \cong \overrightarrow{\lim }_{U} H^{n-p-1}(U) \\
& \cong \begin{cases}0, & \text { if } p \neq n-1 ; \\
\mathbb{Z}, & \text { if } p=n-1 .\end{cases}
\end{aligned}
$$

This completes the proof of the theorem. 
Remarks. Finite cohomological dimension is required, since, for example, a Hilbert cube manifold has trivial homology sheaf (and so on this definition would equal its boundary). Local compactness is also required, as can be seen by removing from the boundary of $B^{n}$ a set $K$ such that $\partial B^{n}-K$ is not a (homology) manifold. The results hold if coefficients for homology and cohomology are taken in any countable PID.

\section{REFERENCES}

[B1] G. E. Bredon, Wilder manifolds are locally orientable, Proc. Nat. Acad. Sci. USA 63 (1969), 1079-1081.

[B2] Sheaf theory, McGraw-Hill, 1967.

[D] A. Dold, Partitions of unity in the theory of fibrations, Ann. of Math. 78 (1963), 223-255.

[Ha] A. É. Harlap, Local homology and cohomology, homological dimension and homology manifolds, Mat. Sbornik 96 (1975), 347-373 (Russian); Math. USSR Sbornik 25 (1975), 329-349.

[Hu] P. J. Huber, Homotopical cohomology and Čech cohomology, Math. Ann. 144 (1961), 73-76.

[M] W. J. R. Mitchell, Homology manifolds, inverse systems and cohomological local connectedness, J. London Math. Soc. (2) 19 (1979), 348-358.

[Ra] F. Raymond, Separation and union theorems for generalized manifolds with boundary, Michigan Math. J. 7 (1960), 7-21.

[Re] D. Repovš, Generalized 3-manifolds with 0-dimensional singular set, Thesis, Tallahassee, 1983.

[S] E. G. Skljarenko, On the theory of generalised manifolds, Izv. Akad. Nauk SSSR Ser. Mat 35 (1971), 831-843 (Russian); Math. USSR Izv. 5 (1971), 845-857.

[Wh] P. A. White, Some characterizations of homology manifolds with boundaries, Canad. J. Math. 4 (1952), 329-342.

[Wi] R. L. Wilder, Topology of manifolds, Amer. Math. Soc. Colloq. Publ., vol. 32, Providence, RI, 1963.

Department of Mathematics, Magdalene College, Cambridge, CB3 0AG, United KingDOM 